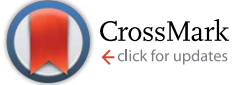

Cite this: RSC Adv., 2016, 6, 2582
Received 12th November 2015 Accepted 21st December 2015

DOI: 10.1039/c5ra23926e

www.rsc.org/advances

\section{On the origin of functionalization in one-pot radiation synthesis of nanogels from aqueous polymer solutions $\uparrow$}

\author{
C. Dispenza, ${ }^{\text {ad }}$ M. A. Sabatino, ${ }^{a}$ N. Grimaldi,,$^{a}$ M. R. Mangione, ${ }^{b}$ M. Walo, ${ }^{c}$ \\ E. Murugan ${ }^{d}$ and M. Jonsson ${ }^{d}$
}

\begin{abstract}
Radiation-engineered poly( $\mathrm{N}$-vinyl pyrrolidone) nanogels are very interesting biocompatible nanocarriers for i.v. administration of therapeutics and contrast agents for bioimaging. The manufacturing process is fast and effective, it grants excellent control of particle size and simultaneous sterilization of the formed nanogels. Interestingly, primary amino groups and carboxyl groups, useful for (bio)conjugation, are also formed in a dose-dependent fashion. In this paper, by means of both numerical simulations and experiments, the origin of nanogel size control and functionalization is investigated. This understanding offers a new dimension for the design and production of radiation-sculptured multifunctional nanocarriers from aqueous solutions of polymers.
\end{abstract}

\section{Introduction}

The radiation chemistry of polymers in solution has been studied quite extensively for several decades. Irradiation of polymers in solution using ionizing radiation leads to inter- and intramolecular crosslinking, chain scission and to alterations of the chemical functionality of the polymers. The relative extent of the different types of alterations is mainly governed by the nature of the polymer and the solvent, polymer concentration, presence of other co-solutes, but also by dose rate and total dose. In particular, the presence of dissolved gases, such as $\mathrm{O}_{2}$ and $\mathrm{N}_{2} \mathrm{O}$, has an impact on the final outcome of the process..$^{\mathbf{1 , 2}}$ Intermolecular crosslinking of concentrated hydrophilic polymers in water leads to the formation of macroscopic networks called macrogels, while intramolecular crosslinking leads to the formation of small polymeric particles known as nanogels. ${ }^{3-11}$ Nanogels are among the few hydrophilic nanoparticles, that are also stable colloids, currently under investigation as drug carriers for intravenous administration. ${ }^{\mathbf{1 2 - 1 5}}$ While radiation-

${ }^{a}$ Dipartimento di Ingegneria Chimica, Gestionale, Informatica, Meccanica, Università degli Studi di Palermo, Viale delle Scienze, Edificio 6, 90128 Palermo, Italy. E-mail: clelia.dispenza@unipa.it; Fax: +39091 2386080; Tel: +39 3204328593

${ }^{b}$ CNR - Istituto di Biofisica (IBF) UOS Palermo, Via U. La Malfa 153, 90146 Palermo, Italy

${ }^{c}$ Centre for Radiation Research and Technology, Institute of Nuclear Chemistry and Technology, Dorodna 16, 03-195 Warsaw, Poland

${ }^{d}$ School of Chemical Science and Engineering, Applied Physical Chemistry, KTH Royal Institute of Technology, SE-100 44 Stockholm, Sweden

$\dagger$ Electronic supplementary information (ESI) available. See DOI: 10.1039/c5ra23926e

\$ Current address: Nanomol Technologies SA, Módul de Recerca B, Campus Universitari de Bellaterra, 08193, Cerdanyola del Vallès, Spain. engineered macrogels are already established in numerous industrial, agricultural, personal care and medical applications, their nanoscale analogues have only recently been proposed as nanocarriers of molecular drugs, ${ }^{\mathbf{1 6 , 1 7}}$ model proteins, ${ }^{\mathbf{1 0}}$ oligonucleotides, ${ }^{18}$ insulin ${ }^{19}$ and decorated with chelating agents for immobilization of paramagnetic ions. ${ }^{20}$ Ongoing research is providing a growing body of evidence that these nanoparticles are biocompatible, hemocompatible and able to be internalized by cells. In order to fully exploit their potential, fundamental understanding of the mechanism of their formation is essential. In dilute or semi-dilute aqueous polymer solutions, the ionizing radiation is primarily absorbed by water, leading to production of oxidizing and reducing species. The primary oxidizing species are $\mathrm{OH}^{-}$and $\mathrm{H}_{2} \mathrm{O}_{2}$, while the primary reducing species are $\mathrm{e}_{\mathrm{aq}}{ }^{-}, \mathrm{H}^{\cdot}$ and $\mathrm{H}_{2}$. The radiation chemical yields $(G-$ values) in gamma and electron irradiated aqueous solutions are $0.28,0.073,0.28,0.062$ and $0.047 \mu \mathrm{mol} \mathrm{J}^{-1}$ for $\mathrm{OH}^{-}, \mathrm{H}_{2} \mathrm{O}_{2}, \mathrm{e}_{\mathrm{aq}}{ }^{-}$, $\mathrm{H}^{\bullet}$ and $\mathrm{H}_{2}$, respectively. For most polymers, only the hydroxyl radical (and the hydrogen atom) is capable of producing the radical precursors for nano- and macro-gel formation, mainly by $\mathrm{H}$-abstraction. By saturating the aqueous solution with $\mathrm{N}_{2} \mathrm{O}$ prior to irradiation, the yield ( $G$-value) of the hydroxyl radical can be doubled since the solvated electron is scavenged by $\mathrm{N}_{2} \mathrm{O}$ to produce hydroxyl radicals. ${ }^{21}$

Radiation manufacturing of nanogels is usually performed using e-beam irradiation from an electron accelerator. Accelerators are operated in pulsed mode. Hence, irradiation is discontinuous and every short pulse of electrons is followed by a relatively long time without irradiation. Radical reactions are induced during the electron pulse but they may not be entirely completed before the next electron pulse is absorbed by the sample. Follow-up chemical reactions will also occur between 
and during pulses. This makes the kinetic analysis of the reaction system more complex than for a system exposed to continuous irradiation.

The general simplification that is often made when analyzing data from pulsed e-beam irradiations of aqueous polymer solutions, is that all hydroxyl radicals produced upon radiolysis of water are scavenged by the polymeric solute to form carbon-centered macroradicals. ${ }^{7,8,22}$ Hence, the average number of macroradical centers formed in a pulse would then simply be determined from the radiation chemical yield of hydroxyl radicals. This may be true at higher polymer concentrations but not necessarily at lower concentrations, where other reactions involving the hydroxyl radical could efficiently compete with the reaction of hydroxyl radicals with the polymer. Even if the concentration in terms of repeating monomer units can be fairly high, the actual polymer concentration is low and thereby the average distance between polymer molecules is large. At high dose rate (as in most cases of pulsed e-beam irradiation), radical-radical combination reactions are favorable and a higher polymer concentration is required to scavenge the hydroxyl radicals formed. If the polymer concentration is not sufficiently high, hydroxyl radical combination will yield hydrogen peroxide and conversion of initially formed hydroxyl radicals to polymer radicals will not be quantitative. Hence, monitoring $\mathrm{H}_{2} \mathrm{O}_{2}$ build-up can be used to understand about how efficiently hydroxyl radicals are converted into polymer radicals at different polymer concentrations. The fact that $\mathrm{H}_{2} \mathrm{O}_{2}$ build-up is possible under certain conditions also means that the chemical environment in the irradiated polymer solution will be different at low and high polymer concentrations, as the $\mathrm{H}_{2} \mathrm{O}_{2}$ concentration is expected to be higher in the more dilute polymer solutions. This may have an impact on the overall process. $\mathrm{H}_{2} \mathrm{O}_{2}$ is also a precursor for $\mathrm{O}_{2}$, which will strongly influence the chemistry of C-centered polymer radicals. To fully understand the mechanism and the kinetics of nanogel formation, it is essential to quantify the primary production of polymer radicals. Complete mechanistic knowledge is also a prerequisite for process optimization.

In this work, we have studied the initial formation of $\operatorname{poly}(\mathrm{N}$ vinyl pyrrolidone) (PVP) radicals using $\mathrm{H}_{2} \mathrm{O}_{2}$ as a probe for the competition between hydroxyl radical recombination and hydroxyl radical reaction with the polymer. On the basis of these results and results on particle size and relative changes in polymer functionality (determined using FTIR and titration) as function of polymer concentration and total dose, the kinetics and mechanism of PVP nanogel formation in irradiated aqueous systems are discussed.

\section{Experimental}

\section{Hydrogen peroxide build-up: experiments and simulations}

The $\mathrm{H}_{2} \mathrm{O}_{2}$ production experiments were performed using a 9 $\mathrm{MeV}$ Microtron electron accelerator delivering $50 \mathrm{~ns}$ pulses at a frequency of $25 \mathrm{~Hz}$. The average dose rate was $20 \mathrm{~Gy} \mathrm{~s}^{-1}$ and the dose rate during the pulse was $1.6 \times 10^{7} \mathrm{~Gy} \mathrm{~s}^{-1}$. The dose rate was determined using the Fricke dosimeter and the ferrouscupric sulfate dosimeter. ${ }^{23} 10 \mathrm{ml} \mathrm{N}_{2} \mathrm{O}$ saturated aqueous solutions were irradiated under the above conditions for different periods of time and the $\mathrm{H}_{2} \mathrm{O}_{2}$ concentration was determined using the Ghormley triiodide method after irradiation. ${ }^{24,25}$ In this method, $\mathrm{H}_{2} \mathrm{O}_{2}$ oxidizes $\mathrm{I}^{-}$to $\mathrm{I}_{3}{ }^{-}$which is quantified using a UV-vis spectrophotometer $(\lambda=302 \mathrm{~nm})$. The reagents used in this method are ammoniumdimolybdate, ADM (Alfa Aesar, 4\% w/v), and potassium iodide, KI (VWR BDH Prolabo, 99.0\%).

Numerical simulations of the system were performed using the software Maksima Chemist. ${ }^{26}$ This software is designed for numerical simulations of homogeneous radiation chemistry. The simulations were based on all reactions involved in the radiolysis of water and their corresponding rate constants and the $G$-values for electron irradiation of water. The dose rates were varied in the simulations in order to investigate the behavior under different irradiation conditions. Simulations were performed for pure water and for aqueous solutions containing variable concentrations of hydroxyl radical scavenger. To mimic the behavior of a polymer, the concentration of hydroxyl radical scavenger was locked, i.e. the concentration remained constant throughout each simulation. This was done to account for the large number of abstractable hydrogen atoms on each polymer molecule, i.e. the high localized scavenging capacity.

\section{Synthesis of PVP nanogels}

PVP was purchased from Aldrich. The polymer has average weigh molecular weight $M_{\mathrm{w}}=6.44 \mathrm{MDa}$, as determined from static light scattering measurements. Polymer critical chain overlapping concentration in water was estimated to be about $0.85 \mathrm{wt} \%$, by intrinsic viscosity measurements.

PVP aqueous solutions with concentrations in the range 0.1$0.5 \mathrm{wt} \%$ were filtered with $0.22 \mu \mathrm{m}$ cut off syringe filters, carefully deoxygenated by gaseous nitrogen, bottled in hermetically closed $15 \mathrm{ml}$ glass vials and saturated with $\mathrm{N}_{2} \mathrm{O}$ prior to irradiation. Electron beam irradiation was performed using Elektronika 10/10, a $10 \mathrm{MeV}$ linear accelerator of the ICHTJ in Warsaw (Poland). Irradiation was carried out at an average beam current of $450 \mathrm{~mA}$, pulse length of $4.5 \mu \mathrm{s}$ and pulse repetition rate of $300 \mathrm{~Hz}$. The dosimetry was performed using a graphite calorimeter with an error of $\pm 4 \%$. Samples have been horizontally placed in a box filled with ice $\left(4-8{ }^{\circ} \mathrm{C}\right)$ and conveyed under the beam via a transporting belt at a speed of $0.15 \mathrm{~m}$ $\min ^{-1}$. An integrated dose of $20 \mathrm{kGy}$ was supplied with a single pass, while higher doses were obtained with a multiple exposure of $20 \mathrm{kGy}$ for each pass. From the measurement of the residence time of the vials under the beam ( $5 \mathrm{~s})$, an approximate absorbed dose per pulse of about 13 Gy has been estimated. After irradiation, samples were dialyzed against distilled water for $48 \mathrm{~h}$ using dialysis tubes of $100 \mathrm{kDa}$ cut-off (Sigma Aldrich). Nanogel systems are coded as $\operatorname{NG}(X) Y$, where $X$ is the concentration of polymer in the irradiated aqueous solution (\% wt) and $Y$ is the total absorbed dose in kGy.

\section{Molecular and physico-chemical characterization of nanogels}

Hydrodynamic diameters $\left(D_{\mathrm{h}}\right)$ of NGs dispersed in bidistilled water (as produced), and after being freeze-dried with 
saccharose (6 wt\%) as cryoprotector and redispersed in $\mathrm{N}$ methyl-pyrrolidone were measured by dynamic light scattering (DLS) measurements. Weight average molecular weight $\left(M_{\mathrm{w}}\right)$ of nanogels were estimated from multi-angle static light scattering (SLS) measurements of the NGs aqueous dispersions at $25^{\circ} \mathrm{C} \pm$ $0.1{ }^{\circ} \mathrm{C}$. Both DLS and SLS were performed using a Brookhaven BI-9000 correlator and a $50 \mathrm{~mW} \mathrm{He}-\mathrm{Ne}$ laser (MellesGriot) tuned at $\lambda=632.8 \mathrm{~nm} .{ }^{11}$ The DLS data were fitted by the method of cumulants. ${ }^{27,28}$

The SLS data were analyzed according to the Zimm plot method, using experimentally measured refractive index increments $\mathrm{d} n / \mathrm{d} c$ values measured by using a Brookhaven Instruments differential refractometer at $\lambda=620 \mathrm{~nm} .{ }^{11}$ Measurements were carried out on a minimum two samples from three independent runs.

Gel filtration chromatography analysis was performed using two Shodex SB HQ columns in series (806 and 804) thermostated at $20{ }^{\circ} \mathrm{C}$ with an Knauer oven, connected to a HPLC device (LC-2010 AT Prominence, Shimadzu, Kyoto, Japan) equipped with a $50 \mu \mathrm{l}$ sample loop. All samples were eluted with $0.02 \mathrm{wt} \%$ sodium azide solution at $0.5 \mathrm{ml} \mathrm{min}^{-1}$; the refractive index was recorded with a Smartline RI detector 2300 Knauer.

Atomic force microscopy (AFM) was performed on selected aqueous NGs systems ( $5 \mathrm{ng} \mathrm{ml}^{-1}$ ) deposited on a mica substrate modified with 3-aminopropyl triethoxysilane (APTES). ${ }^{29}$ Samples have been examined under water in tapping mode at 2 Hz scanning rate using a MMFP3D AFM (Asylum Research) and MSNL D tips (Bruker Nano Inc.) with nominal resonance frequency of $10-20 \mathrm{kHz}$ and spring constant $k \sim 30 \mathrm{pN} \mathrm{nm}^{-1}$.

\section{Chemical structure characterization of nanogels}

Chemical modification occurring upon irradiation was assessed by FTIR and colorimetric titration. More in detail, FTIR analysis was carried out with Perkin Elmer-Spectrum 400 apparatus by dispersing the dry product in potassium bromide and compressing into pellets. Spectra were recorded at 30 scans per spectrum and $2 \mathrm{~cm}^{-1}$ resolution in the $4000-450 \mathrm{~cm}^{-1}$ range. All spectra have been normalized with respect to the peak correspondent to the stretching of methylene groups (2956 $\mathrm{cm}^{-1}$ ). Quantification of functional groups formed upon irradiation, such as carboxyl groups and primary amino groups was performed by titration. In particular, reaction of primary amino groups with fluorescamine produces a fluorescent adduct with a maximum emission at $480 \mathrm{~nm}$ (excitation wavelength of 392 $\mathrm{nm}) \cdot{ }^{30}$ Dialyzed nanogel samples were diluted with water to the final concentration of $0.25 \mathrm{mg} \mathrm{ml}^{-1}$, added with an equal volume of fluorescamine/acetonitrile solution $\left(2.5 \mathrm{mg} \mathrm{ml}^{-1}\right)$ and twofold diluted with $\mathrm{PBS} \mathrm{pH}$ 7.4. The reaction is performed at ambient temperature for 15 min under stirring in dark conditions. Fluorescence spectra were recorded immediately after, on a FP-8200 Spectrofluorometer (Jasco). A calibration curve was built using aminopropylacrylamide solutions reacted with fluorescamine at different concentrations.

NGs carboxyl groups were quantified by using their ability to extract $\mathrm{Ni}^{2+}$ cations from an aqueous solution. ${ }^{31} \mathrm{~A}$ given volume of PVP-based NGs was mixed with $\mathrm{Ni}^{2+} /$ Hepes solution $\mathrm{pH} 7.5$ (total volume $2 \mathrm{ml}, \mathrm{Ni}^{2+}$ concentration $20 \mu \mathrm{M}$ ) for $2 \mathrm{~min}$. After centrifugation for $30 \mathrm{~min}$ at $3000 \mathrm{rpm}$, using 100k MWCO Vivaspin-2 (Sartorius) filters, $1 \mathrm{ml}$ of the filtrated product was collected and diluted to the final volume of $2 \mathrm{ml}$ with $40 \mu \mathrm{M}$ pyrocatechol violet (PV) in Hepes solution (freshly prepared on a daily basis).

Absorption spectra were recorded immediately after PV addition. The absorbance at $650 \mathrm{~nm}$ was measured for the determination of free $\mathrm{Ni}^{2+}$. The amount of $\mathrm{Ni}^{2+}$ sequestered by nanogels was determined as the difference between the amount of nickel initially added to the nanogels solution and free $\mathrm{Ni}^{2+}$ bound to PV.

\section{Results and discussion}

\section{Macroradical vs. $\mathrm{H}_{2} \mathrm{O}_{2}$ formation}

Prior to the experiments using $\mathrm{H}_{2} \mathrm{O}_{2}$ build-up to probe the efficiency of converting hydroxyl radicals to polymer radicals, we performed a numerical simulation of the reactions system. The simulation was performed using MACKSIMA CHEMIST and the pulse sequence was mimicked by alternately performing the simulation for the pulse and for the time between pulses using the final concentrations of all species from the previous simulation as input data for next simulation. ${ }^{26}$ The results are shown in Fig. 1. As can be seen, $\mathrm{H}_{2} \mathrm{O}_{2}$ build-up takes place during and immediately after the electron pulse. Not surprisingly, the presence of a radical scavenger reduces the build up of $\mathrm{H}_{2} \mathrm{O}_{2}$. To illustrate the behavior of the system at higher doses (higher number of pulses), we also performed a simulation using continuous irradiation with the same dose rate as during the pulse. In this case the simulations were performed at a number of different scavenger concentrations.

Since a macromolecule has a very high scavenging capacity, the simulations were performed using locked scavenger (i.e. polymer) concentration, i.e., the scavenging capacity does not change during the irradiation (the scavenger is not consumed).

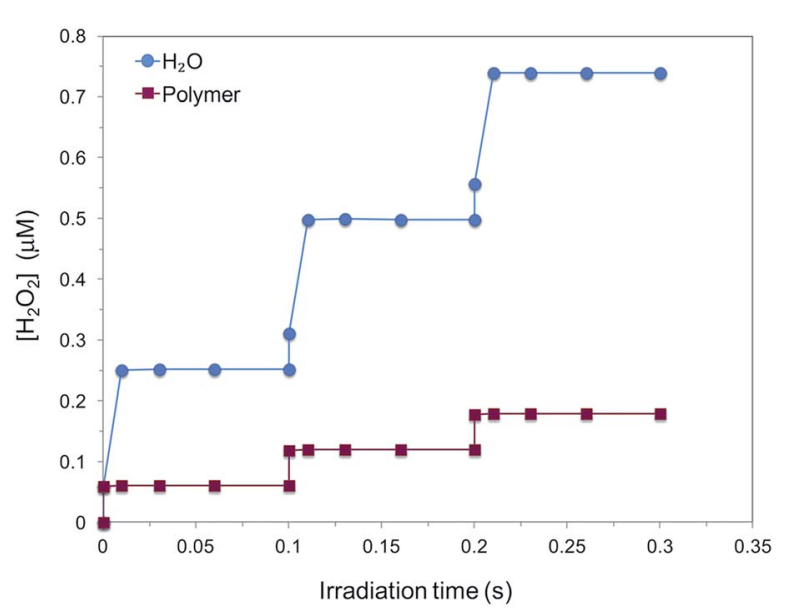

Fig. $1 \mathrm{H}_{2} \mathrm{O}_{2}$ concentration as a function of reaction time for pure water and for water containing $10 \mathrm{mM}$ of hydroxyl radical scavenger during a sequence of three pulses. Results obtained from numerical simulations. 
This assumption is not valid for low molecular weight scavengers (unless the conversion is low) and only valid up to a certain point for macromolecules. The results are shown in Fig. 2. We can see that a significant reduction in $\mathrm{H}_{2} \mathrm{O}_{2}$ production is only observed at scavenger concentrations of about $0.1 \mathrm{mM}$ and above. This implies that a quantitative conversion of hydroxyl radicals to polymer radicals only occurs above a "critical" polymer concentration.

The results from the e-beam experiments using $\mathrm{H}_{2} \mathrm{O}_{2}$ formation as a probe for the efficiency of polymer radical formation are presented in Fig. 3. Irradiations were performed with pure water and polymer aqueous solutions at two concentrations, 0.05 and $0.5 \%$ wt. For comparison, irradiations in the presence of a low molecular weight scavenger, $\left(\mathrm{CH}_{3}\right)_{3} \mathrm{COH}$, at the same concentrations (in wt\%) as for the polymer, were also performed. As can be seen, the $\mathrm{H}_{2} \mathrm{O}_{2}$ concentration increases significantly with irradiation time for pure water. The plot is qualitatively in agreement with the simulation: the $\mathrm{H}_{2} \mathrm{O}_{2}$ concentration appears to approach a steady-state value; we can also observe that the presence of both high and low molecular weight hydroxyl radical scavengers results in reduced $\mathrm{H}_{2} \mathrm{O}_{2}$ production in the system.

For the polymer solutions it is evident that the lowest concentration is not sufficient to completely prevent $\mathrm{H}_{2} \mathrm{O}_{2}$ formation. This clearly shows that the hydroxyl radicals are not quantitatively converted into polymer radicals at this polymer concentration. It is also interesting to note that $\left(\mathrm{CH}_{3}\right)_{3} \mathrm{COH}$ is significantly more efficient in reducing the $\mathrm{H}_{2} \mathrm{O}_{2}$-formation at the same weight\% as the polymer.

In practice, semi-dilute polymer solutions should be regarded as heterogeneous systems where the concentration of repeating monomer units is locally very high (for the volume elements containing the polymer) and where there are volume elements containing no repeating monomer units. The hydroxyl radical scavenging capacity is only expressed in the volume elements containing the polymer. For the solutions containing low molecular weight scavengers the situation is completely

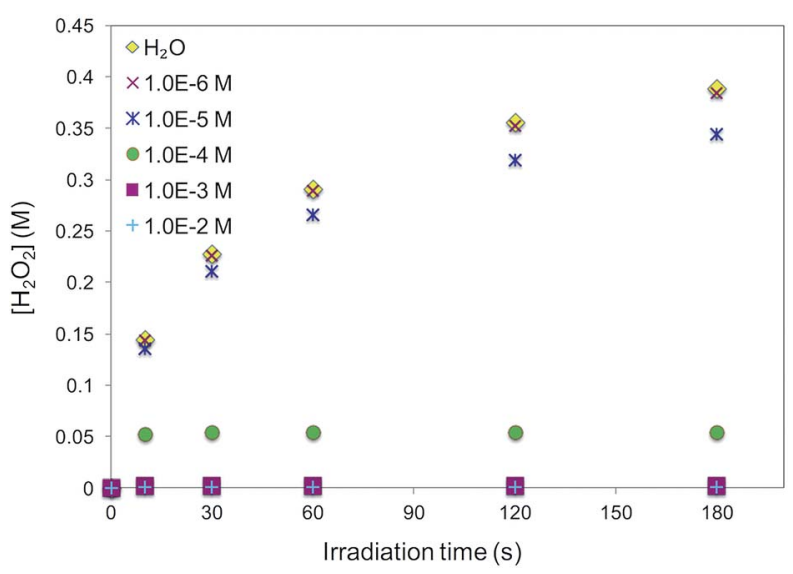

Fig. $2 \mathrm{H}_{2} \mathrm{O}_{2}$ concentrations as function of irradiation time for pure water and for aqueous solutions containing various concentrations of hydroxyl radical scavengers during continuous irradiation at the dose rate during the pulse (16 MGy s${ }^{-1}$ ). Results obtained from numerical simulations.

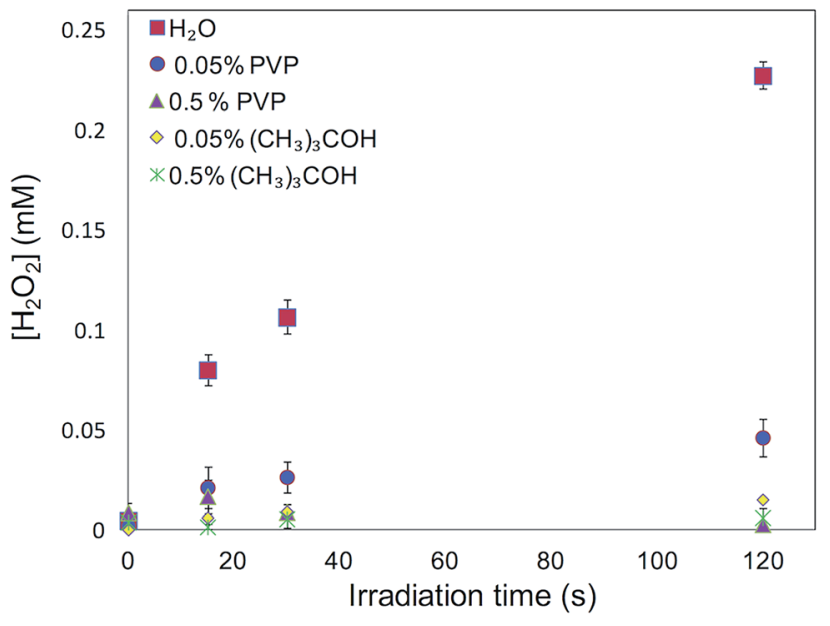

Fig. $3 \quad \mathrm{H}_{2} \mathrm{O}_{2}$ concentration as a function of irradiation time at a pulse length of $50 \mathrm{~ns}$ and a frequency of $25 \mathrm{~Hz}$ for $\mathrm{N}_{2} \mathrm{O}$ saturated water and aqueous solutions containing PVP and $\left(\mathrm{CH}_{3}\right)_{3} \mathrm{COH}$.

different. Here, the system is homogeneous and the scavenging capacity at the given weight\% is much higher. Hence, molecular concentration in moles per liter is the most relevant means of comparing scavenging capacity in systems of both low molecular weight and high molecular weight systems. The obvious conclusion from this is that the total polymer radical concentration formed in a pulse will increase with increasing polymer concentration until full scavenging capacity is achieved and before this condition is achieved, $\mathrm{H}_{2} \mathrm{O}_{2}$ formation has to be considered. Taking into account the heterogeneity of the system and the locally high hydroxyl radical scavenging capacity, we can also conclude that the number of radical centers produced per polymer molecule will be the same up to the polymer concentration where full scavenging capacity is achieved. Up to this point the rate constant for intramolecular radical crosslinking will be concentration independent as will the rate constant for intermolecular crosslinking (same distribution of radicals). However, as the rate of intermolecular crosslinking depends on the square of the concentration, the competition between intra- and intermolecular crosslinking will change in favor of intermolecular crosslinking with increasing polymer concentration. Above the concentration where full scavenging capacity is achieved, the number of radicals produced per polymer molecule will decrease with increasing polymer concentration, the total number of macroradical centers formed in a pulse remaining constant. As a result, the average number of independent radical carriers, i.e. macromolecules carrying radicals, will increase with increasing polymer concentration, provided that the polymer concentration does not exceed the number of radicals formed in the pulse.

In this concentration range (i.e. above full scavenging capacity) the rate constants for both intra- and intermolecular crosslinking will change and eventually the rate constant for intramolecular crosslinking will become zero at the polymer concentration where one or less macroradical center per polymer molecule is obtained. Under these conditions only intermolecular crosslinking is possible. 


\section{Physico-chemical properties of PVP nanogels}

Poly( $N$-vinyl pyrrolidone) aqueous solutions at various concentrations, in the range $0.1-0.5 \mathrm{wt} \%$, have been irradiated providing a total dose of $20 \mathrm{kGy}$ in a single pass. Higher doses were obtained by multipass exposure. All the systems appeared optically transparent after irradiation and increasing yellow in color at the decrease of polymer concentration. They were characterized through both dynamic and static light scattering for the determination of the hydrodynamic diameter and weight average molecular weight, respectively. Data are shown in Table 1. A monotonic increase of molecular weight of the systems at the increase of polymer concentration above $0.1 \mathrm{wt} \%$ is evident.

On the contrary, the hydrodynamic diameter of the nanoparticles is smaller than that of the non-irradiated polymer when obtained from concentrations lower than $0.3 \mathrm{wt} \%$, and it becomes increasingly larger with the increase of concentration above this value. These results confirm the common description of these systems as the result of combined effects of both intramolecular and inter-molecular crosslinking due to macroradical combination reactions, with the dominance of the former in dilute polymer solutions. ${ }^{3,11}$

Judging from the molecular weight, no significant intermolecular crosslinking is occurring at $0.1 \mathrm{wt} \%$, as well as no molecular degradation (the weight average molecular weight does not change). Consequently, this polymer concentration is sufficiently low to favor intra-molecular crosslinking.

Fig. 4 shows the elution profiles, related to molecular weight distributions, of the nanogels and non irradiated polymer, as reference. The starting polymer is highly polydisperse, with two main populations at high molecular weight (low volumes of retention) and a long tail of low molecular weight fractions. This tail is no longer present in all the irradiated systems, irrespective of concentration.

All the irradiated systems are multimodal but significantly less polydisperse than the linear polymer. A distinct shift of the refractive index signal toward the high molecular weights (smaller elution volumes) is observed at the increase of concentration from 0.1 to $0.5 \mathrm{wt} \%$. For the intermediate concentrations, the dimensions of the higher molecular weight families increase by partially depleting the lower molecular weight families, as if their growth occurred by aggregation of some "primary" nanoparticles. These primary nanoparticles appear to be formed by intermolecular crosslinking of shorter

Table 1 Weight average molecular weight and hydrodynamic diameters in water for nanogels obtained at 20 kGy varying the polymer concentration

\begin{tabular}{lcc}
\hline System & $M_{\mathrm{w}}\left(\mathrm{g} \mathrm{mol}^{-1} \times 10^{5}\right)$ & $D_{\mathrm{h}}(\mathrm{nm})$ \\
\hline PVP non-irr & $6.44 \pm 0.3$ & $33.4 \pm 10.5$ \\
NG(0.1)20 & $6.38 \pm 0.5$ & $21.2 \pm 6.7$ \\
NG(0.2)20 & $17.4 \pm 0.1$ & $19.3 \pm 9.6$ \\
NG(0.3)20 & $41.0 \pm 1.0$ & $38.5 \pm 17.1$ \\
NG(0.4)20 & $126 \pm 7.0$ & $53.7 \pm 27.1$ \\
NG(0.5)20 & $680 \pm 18.0$ & $115.6 \pm 39.8$
\end{tabular}

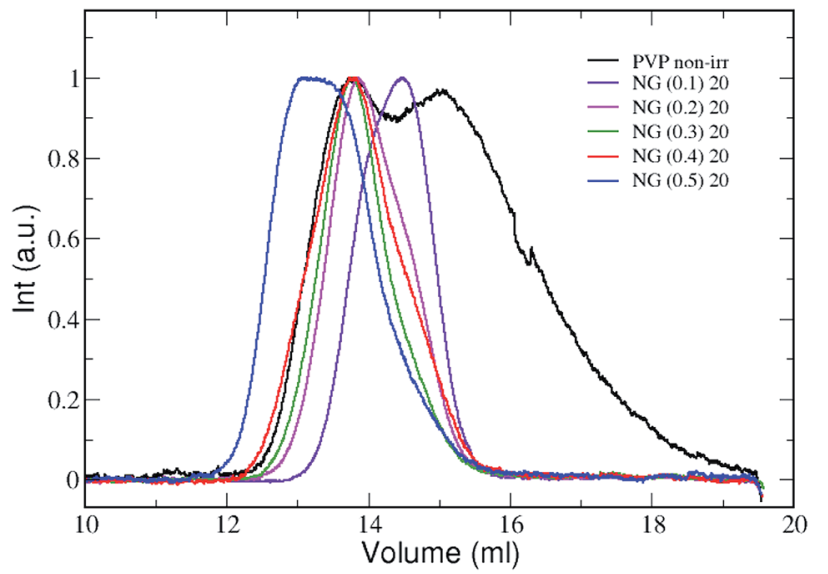

Fig. 4 Chromatographic profiles for the nanogels produced at $20 \mathrm{kGy}$, varying the polymer concentration. The GFC curve for the non-irradiated polymer is also included as reference.

length chains (from the disappearance of the low molecular weight tails) as well as intramolecular crosslinking of the longer length chains (from the disappearance of the high molecular weight tails at low concentration). These effects can be better appreciated by applying a Gaussian deconvolution to the curves, which is shown in Fig. S1 of the ESI. $\dagger$ In particular, the chromatographic profiles of the $\mathrm{NG}(0.2), \mathrm{NG}(0.3)$ and $\mathrm{NG}(0.4)$ nanogels are well described by three families. The change of percentage area of the three families is shown in Table. $S 1 . \dagger$ The percentage area of the first family, corresponding to the objects of smallest size, gradually decreases while that of the second, for $\mathrm{NG}(0.3)$, or the third family, for $\mathrm{NG}(0.4)$, increase.

The narrowing of the molecular weight distribution upon irradiation can be discussed in view of the results on the scavenging capacity presented above.

The low molecular weight fraction will have a higher hydroxyl radical scavenging capacity per unit weight simply because there will be more polymer chains per unit weight, and they will tend to form fewer radicals per chain due to the lower number of abstractable hydrogen atoms compared to polymer chains of average size.

This will favor intermolecular cross-linking of smaller macromolecules to form larger networks. In contrast, polymer chains with sizes above the average size will tend to form more radicals per chain, which will favor intra-molecular crosslinking and shrinkage of polymer coils.

As a result, the nanogel size distribution is significantly narrower than the size distribution of the parent linear polymer coils.

Polymer solutions at the two extreme concentrations $(0.1$ and $0.5 \mathrm{wt} \%$ ) have been irradiated at increasing doses, by multiple passes under the electron beam. In Table 2 data obtained from DLS and SLS analysis are reported. No significant effects of the total absorbed dose on the average hydrodynamic size and molecular weight are observed, except for a slight contraction of hydrodynamic size of the $\mathrm{NG}(0.1)$ systems up to $60 \mathrm{kGy}$, followed by a slight increase at $80 \mathrm{kGy}$. The elution profiles shown in Fig. 5a and $\mathrm{b}$ evidence only little 
Table 2 Weight average molecular weight and hydrodynamic diameters in water for nanogels obtained at 0.1 and $0.5 \mathrm{wt} \%$, varying the irradiation dose

\begin{tabular}{lcc}
\hline System & $M_{\mathrm{w}}\left(\mathrm{g} \mathrm{mol}^{-1} \times 10^{5}\right)$ & $D_{\mathrm{h}}(\mathrm{nm})$ \\
\hline $\mathrm{NG}(0.1) 20$ & $6.38 \pm 0.51$ & $21.2 \pm 6.7$ \\
$\mathrm{NG}(0.1) 40$ & $6.40 \pm 0.25$ & $16.7 \pm 5.9$ \\
$\mathrm{NG}(0.1) 60$ & $6.80 \pm 0.32$ & $15.0 \pm 5.8$ \\
$\mathrm{NG}(0.1) 80$ & $6.35 \pm 0.10$ & $18.3 \pm 5.0$ \\
$\mathrm{NG}(0.5) 20$ & $680 \pm 18.0$ & $115.6 \pm 39.8$ \\
$\mathrm{NG}(0.5) 40$ & $710 \pm 16.0$ & $115.6 \pm 34.4$ \\
$\mathrm{NG}(0.5) 60$ & $780 \pm 90.0$ & $115.6 \pm 34.4$ \\
$\mathrm{NG}(0.5) 80$ & $750 \pm 105$ & $136.6 \pm 48.1$
\end{tabular}
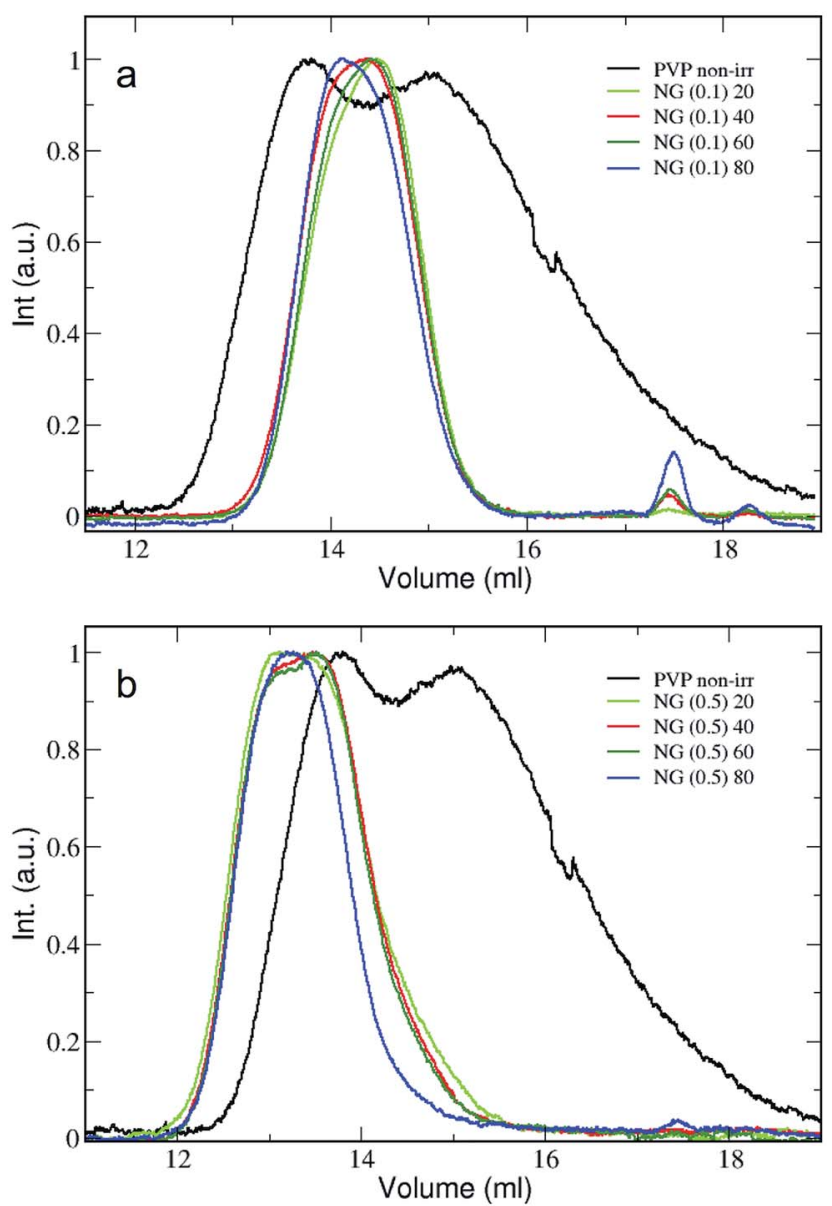

Fig. 5 ( $a$ and $b)$ Chromatographic profiles for the nanogels produced at $0.1 \%$ wt (a) and $0.5 \%$ wt (b), varying the total dose. The GFC curve for the non-irradiated polymer is also included as reference.

modifications of the distributions at the increase of the dose. It can be concluded that, as far as size and molecular weight are concerned, most of the changes have already occurred within the first $20 \mathrm{kGy}$. This result is in good agreement with other studies, which show evidence of nanogels formation at doses of 5-10 kGy. ${ }^{3}$ Beyond $20 \mathrm{kGy}$, the two dominating reactions, intermolecular crosslinking for $\mathrm{NG}(0.1)$ systems and intramolecular for $\mathrm{NG}(0.5)$ systems become both quite ineffective; intramolecular crosslinking increases the rigidity polymer chain segments, thus impairing the formation of further loops; intermolecular crosslinking reduces the polymer concentration and increases the molecular weight, making encounters of radicals in different chains less probable.

In fact, the particle concentration in the $\mathrm{NG(0.5)-system}$ irradiated with $20 \mathrm{kGy}$ is 20 times lower than the particle concentration in the $\mathrm{NG}(0.1)$ system irradiated with the same dose (judging from molecular weights).

A confirmation of the covalent nature of these nanoconstructs is provided by DLS analysis of selected systems in different solvents. In particular, $\mathrm{NG}(0.1)$ and $\mathrm{NG}(0.5)$ obtained upon irradiation at 20 and $80 \mathrm{kGy}$ are freeze-dried and then redispersed using $N$-methyl pyrrolidone (NMP) as solvent to the corresponding original concentration. These systems are also further diluted with water (to 50 vol\% NMP) and compared to the original NG dispersions (non freeze dried) diluted with NMP (to 50 vol\% NMP). The calculated hydrodynamic diameters are reported in Table 3 . In general, the presence of NMP as dispersing medium increases the hydrodynamic diameter of the nanogels and more significantly when NMP is mixed with water. The same effect is achieved by adding NMP to the aqueous dispersions. The organic polar molecule used as a suspending/swelling medium can weaken water-mediated hydrogen bonds and ionic interactions between functional groups in irradiated PVPs. In addition, the presence of a substantially large and nearly planar non-polar region in NMP may result in hydrophobic interactions with the polymer. ${ }^{32}$ Interestingly, the same trend with concentration and dose is followed when the nanogels are dispersed in water, NMP or their mixture.

Finally, a globular yet flexible structure is supported by tapping mode atomic force microscopy in water. AFM images for the 0.1 and $0.5 \mathrm{wt} \%$ systems irradiated at $40 \mathrm{kGy}$ are shown in Fig. $6 \mathrm{a}$ and b. Attractive interactions with the solid substrate induce the round-shaped nanogels to flatten.

\section{Nanogels functionalization}

FTIR analysis provides new interesting elements for the understanding of the formation and evolution of the systems. The most noticeable effects are shown by the NG(0.1) systems irradiated at various doses. Spectra, reported in Fig. 7a and b,

Table 3 Hydrodynamic diameters for nanogels obtained for 0.1 and $0.5 \mathrm{wt} \%$ at 20 and $80 \mathrm{kGy}$, in various solvents

\begin{tabular}{llrll}
\hline System & $\begin{array}{l}D_{\mathrm{h}}(\mathrm{nm}) \\
\text { water }\end{array}$ & \multicolumn{1}{l}{$\begin{array}{l}D_{\mathrm{h}}(\mathrm{nm}) \\
\mathrm{NMP}^{a}\end{array}$} & $\begin{array}{l}D_{\mathrm{h}}(\mathrm{nm}) \\
\mathrm{NMP}^{a}+\text { water }\end{array}$ & $\begin{array}{l}D_{\mathrm{h}}(\mathrm{nm}) \\
\text { water }+\mathrm{NMP}\end{array}$ \\
\hline $\mathrm{PVP}$ & $33.4 \pm 10.5$ & $46 \pm 21.2$ & $133.5 \pm 50.2$ & $130.4 \pm 49.2$ \\
$\mathrm{NG}(0.1) 20$ & $21.2 \pm 6.7$ & $44.4 \pm 14.2$ & $123.2 \pm 41.2$ & $136.2 \pm 50.3$ \\
$\mathrm{NG}(0.1) 80$ & $18.3 \pm 5.0$ & $47.5 \pm 13.4$ & $120 \pm 44.4$ & $130.5 \pm 38.4$ \\
NG(0.5)20 & $115.6 \pm 39$ & $129.3 \pm 38.5$ & $249 \pm 78.3$ & $290.4 \pm 90.3$ \\
NG(0.5)80 & $136.6 \pm 48.1$ & $146.7 \pm 49.2$ & $313 \pm 113.3$ & $330.2 \pm 120.7$
\end{tabular}

${ }^{a}$ Samples redispersed in NMP from the solid state in the presence of saccharose (6 wt\%). 

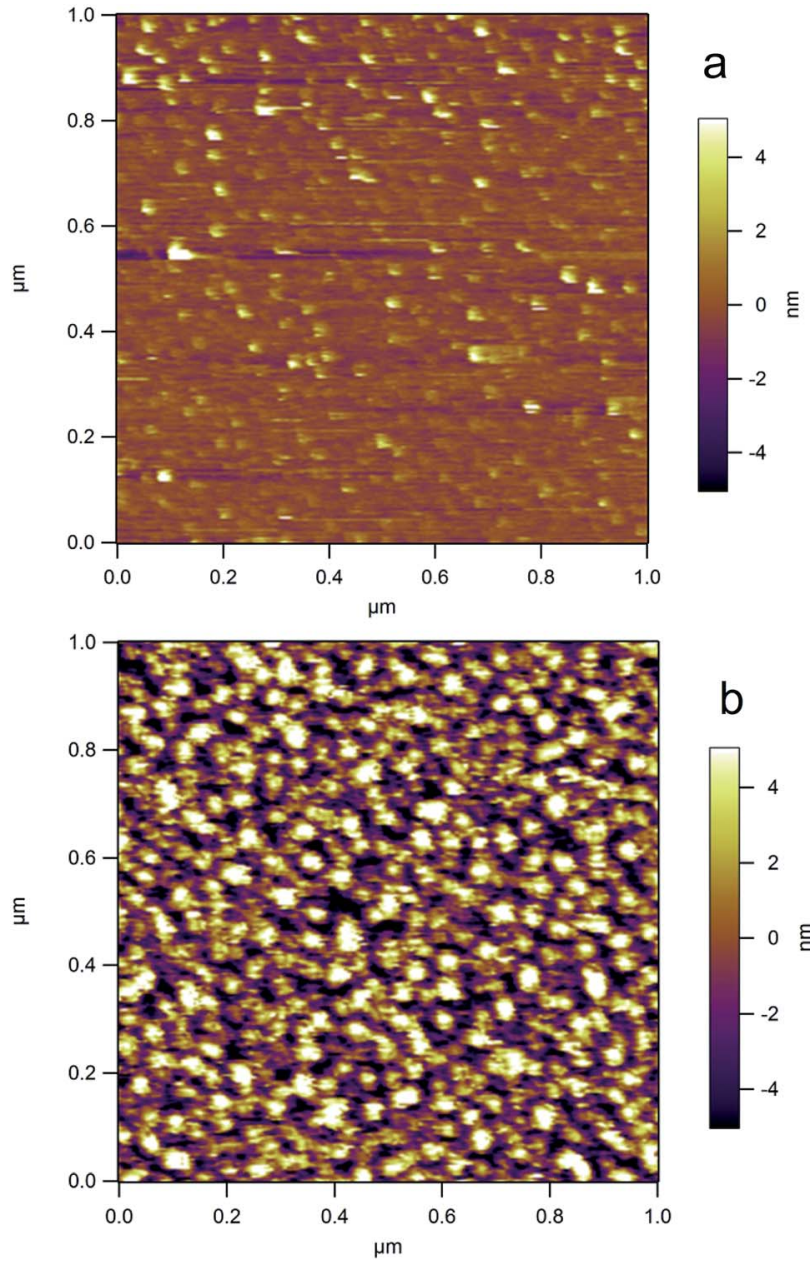

Fig. 6 ( $a$ and b) Height profiles from tapping mode AFM under water show for the $N G(0.1) 40$, (a), and $N G(0.5) 40$, (b). The color scale represents a maximum height of $8 \mathrm{~nm}$.

are normalized with respect to peak at $2956 \mathrm{~cm}^{-1}$, which refers to the stretching of methylene bonds. The spectra of the irradiated systems show increased absorptions in the region 3800$3200 \mathrm{~cm}^{-1}$, that comprises the overlapping contribution of $\mathrm{O}-\mathrm{H}$ stretching vibrations, due residual free and bound water, polymeric hydroxyl groups (the latter in $3400-3200 \mathrm{~cm}^{-1}$ region), and free and associated $\mathrm{N}-\mathrm{H}$ stretching vibrations.

A long tail, overlapping to the characteristic methylene band, stretches up to $2500 \mathrm{~cm}^{-1}$ and it can be attributed to hydroxyl to carbonyl coupling $\left(\mathrm{O}-\mathrm{H}-\mathrm{O}=\mathrm{C}^{\prime}\right)$ of $\mathrm{COOH}$-groups. The presence of carboxyl group dimers is also supported by the characteristic absorption at $1400 \mathrm{~cm}^{-1} \cdot{ }^{33}$ At the increase of dose the concentration of $\mathrm{OH}$-groups remains almost constant and decreases at the highest dose ( $80 \mathrm{kGy})$, while the contribution from $\mathrm{COOH}$-groups steadily increases. Magnification of the spectra in the $1850-1300 \mathrm{~cm}^{-1}$ region (see Fig. $7 \mathrm{~b}$ ) evidences a progressive modification of carbonyl band with the dose. The characteristic carbonyl group vibration of the pyrrolidone group at $1660 \mathrm{~cm}^{-1}$ is the only component of the band for the nonirradiated polymer. At $20 \mathrm{kGy}$, the peak at $1660 \mathrm{~cm}^{-1}$ slightly increases in height and significantly enlarges towards
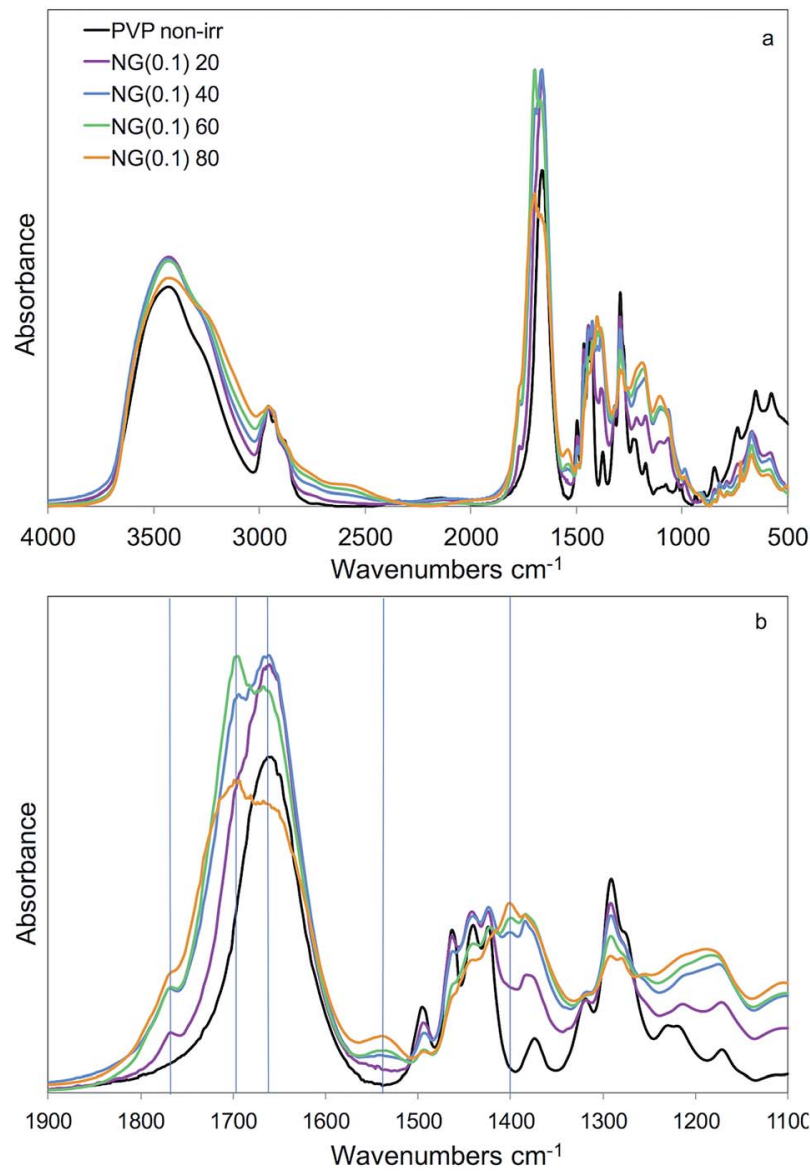

Fig. 7 (a and b) FTIR spectra of NG(0.1) systems irradiated at various doses: (a) full spectra; (b) enlargement in the $1900-1100 \mathrm{~cm}^{-1}$ wavenumbers range.

the higher wavenumbers for the contribution of two new bands, at 1769 and $1698 \mathrm{~cm}^{-1}$, which altogether point to the formation of succinimide rings. The slight enlargement of the band towards the lower wavenumbers can also be due to hydrogen bonding of carbonyl or carboxyl groups with water. Indeed, the frequency of the carbonyl or carboxyl groups with water. Indeed, the frequency of the carbonyl stretching vibration band is very sensitive to hydrogen bond formation. The new peak at 1540 $\mathrm{cm}^{-1}$ is attributed to $\mathrm{N}-\mathrm{H}$ bending vibrations. The relative amount of $\mathrm{N}-\mathrm{H}$ groups and succinimide rings, as well as carboxyl groups, which were not present in the non-irradiated PVP, increases with the dose.

FTIR spectra of nanogels obtained from irradiation of solutions at higher concentration $(0.5 \% \mathrm{wt})$ are reported in Fig. $8 \mathrm{a}$ and $b$. An attenuation of the hydroxyl band is observed for the lower dose $(20 \mathrm{kGy})$, followed by its increase at the increase of the dose. The $\nu(\mathrm{C}=\mathrm{O})$ band steadily increases with dose, it also stretches to the higher wavenumbers and the small new peak at $1769 \mathrm{~cm}^{-1}$ appears. The increase of absorbance of the functional groups that were already present in the non-irradiated polymer, such hydroxyl groups and pyrrolidone carbonyl groups, can be the reflex of methylene groups depletion due to carbon-carbon crosslinking, since the methylene absorption 

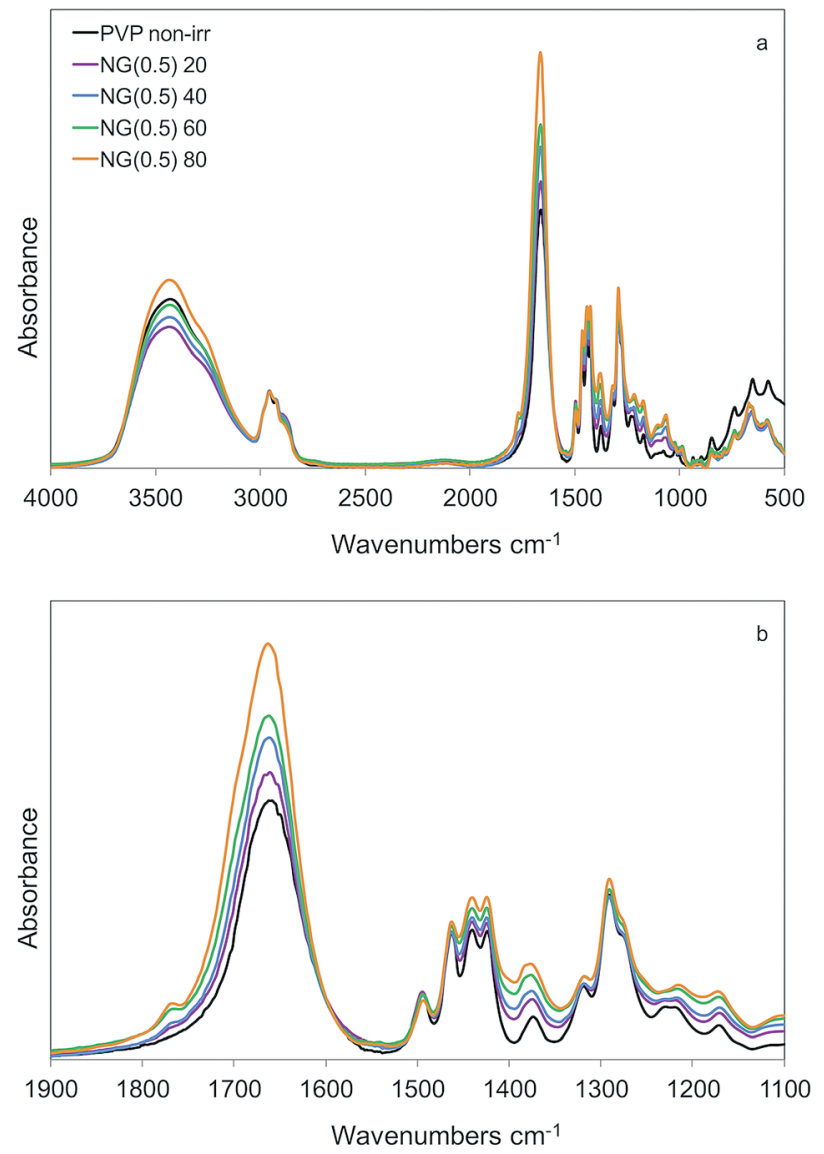

Fig. 8 ( $a$ and b) FTIR spectra of NG(0.5) systems irradiated at various doses: (a) full spectra; (b) enlargement in the $1900-1100 \mathrm{~cm}^{-1}$ wavenumbers range.

was used to normalize the spectra. A partial transformation of pyrrolidone rings in succinimide rings is also visible for these systems, while the presence of carboxyl groups and amino groups is less evident.

In order to quantify carboxyl and amino groups formed upon irradiation, titrations with fluorescamine and $\mathrm{Ni}^{2+}$ were carried out. In particular, fluorescamine reacts only with the primary amino groups, as reported in the literature and confirmed by preliminary tests made with a variety of primary, secondary and tertiary amines. ${ }^{29}$

As can be seen from Table 4, the amount of both $\mathrm{NH}_{2}$ - and $\mathrm{COOH}$-groups increase with increasing dose at a given polymer concentration. It is interesting to note that while size and molecular weight do not change significantly for doses above 20 $\mathrm{kGy}$, the degree of functionalization of the nanoparticles increase with dose beyond this value. For the nanoparticles formed at $0.1 \% \mathrm{PVP}$, the numbers given in Table 3 correspond to $30 \mathrm{NH}_{2}$ - and $100 \mathrm{COOH}$-groups per nanoparticle, for the dose of $80 \mathrm{kGy}$.

In Fig. 9(a and b), the number of functional groups per repeating unit as function of the dose is presented for 0.1 and $0.5 \%$ PVP-solutions. As can be seen, the number of functional groups (both $\mathrm{NH}_{2}$ and $\mathrm{COOH}$ ) per repeating unit increases linearly with dose at both PVP concentrations, the corresponding radiation chemical yields for their formation being in the range of $\mathrm{nmol} \mathrm{J}^{-1}$. At the lower polymer concentration, it is evident that the dose dependence differs for the two types of functional groups; the increase in $\mathrm{NH}_{2}$ concentration with dose is small compared to the increase in $\mathrm{COOH}$ concentration. At the higher polymer concentration, the dose dependence is roughly the same for both types of functional groups. This indicates that the $\mathrm{NH}_{2}$-formation process is kinetically hindered at low polymer concentration. This is also observed when analyzing the effect of polymer concentration on the production of functional groups at $20 \mathrm{kGy}$. As expected, the number of functional groups per repeating unit decreases with increasing polymer concentration at a given absorbed dose. The only exception is the $\mathrm{NH}_{2}$-group density for the $0.1 \mathrm{wt} \% \mathrm{PVP}$, which is virtually identical to the $\mathrm{NH}_{2}$-group density for the $0.2 \mathrm{wt} \%$ PVP. The deviation from the general trend again indicates a limitation in $\mathrm{NH}_{2}$-group formation at the lowest polymer concentration. This limitation leads to an imbalance between $\mathrm{NH}_{2}$ - and $\mathrm{COOH}$-groups for dilute systems at doses above 20 kGy. If desirable, this functionality imbalance can be tailored for the application.

Table 4 Primary amino groups and carboxyl groups formed on PVP upon irradiation, reported both as moles per grams of polymer and moles per moles of repeating units in the polymer. Measured $\mathrm{pHs}$ of nanogel dispersions

\begin{tabular}{|c|c|c|c|c|c|}
\hline System & $\mathrm{NH}_{2} / \mathrm{PVP}\left(\mathrm{g}^{-1}\right)$ & $\mathrm{NH}_{2} / \mathrm{PVP} \mathrm{RU}$ & $\mathrm{COOH} / \mathrm{PVP}\left(\mathrm{g}^{-1}\right)$ & COOH/PVP RU & $\mathrm{pH}$ \\
\hline PVP non-irr & 0 & 0 & 0 & 0 & 6.8 \\
\hline $\mathrm{NG}(0.1) 20$ & $4.1 \times 10^{-5}$ & $4.5 \times 10^{-3}$ & $8.6 \times 10^{-5}$ & $9.4 \times 10^{-3}$ & 5.60 \\
\hline $\mathrm{NG}(0.1) 40$ & $4.1 \times 10^{-5}$ & $4.5 \times 10^{-3}$ & $1.3 \times 10^{-4}$ & $1.5 \times 10^{-2}$ & 4.90 \\
\hline $\mathrm{NG}(0.1) 60$ & $4.6 \times 10^{-5}$ & $5.1 \times 10^{-3}$ & $1.8 \times 10^{-4}$ & $2.0 \times 10^{-2}$ & 4.52 \\
\hline $\mathrm{NG}(0.1) 80$ & $5.3 \times 10^{-5}$ & $5.8 \times 10^{-3}$ & $1.9 \times 10^{-4}$ & $2.0 \times 10^{-2}$ & 4.50 \\
\hline $\mathrm{NG}(0.5) 20$ & $1.5 \times 10^{-5}$ & $1.6 \times 10^{-3}$ & $1.6 \times 10^{-5}$ & $1.8 \times 10^{-3}$ & 6.62 \\
\hline $\mathrm{NG}(0.5) 40$ & $1.8 \times 10^{-5}$ & $2.0 \times 10^{-3}$ & $1.6 \times 10^{-5}$ & $1.8 \times 10^{-3}$ & 6.25 \\
\hline $\mathrm{NG}(0.5) 60$ & $2.4 \times 10^{-5}$ & $2.7 \times 10^{-3}$ & $2.6 \times 10^{-5}$ & $2.9 \times 10^{-3}$ & 5.52 \\
\hline $\mathrm{NG}(0.5) 80$ & $2.8 \times 10^{-5}$ & $3.2 \times 10^{-3}$ & $3.4 \times 10^{-5}$ & $3.8 \times 10^{-3}$ & 5.05 \\
\hline $\mathrm{NG}(0.2) 20$ & $4.1 \times 10^{-5}$ & $4.5 \times 10^{-3}$ & $3.2 \times 10^{-5}$ & $3.5 \times 10^{-3}$ & 6.23 \\
\hline $\mathrm{NG}(0.3) 20$ & $2.1 \times 10^{-5}$ & $2.3 \times 10^{-3}$ & $2.0 \times 10^{-5}$ & $2.2 \times 10^{-3}$ & 6.60 \\
\hline $\mathrm{NG}(0.4) 20$ & $1.6 \times 10^{-5}$ & $1.7 \times 10^{-3}$ & $1.7 \times 10^{-5}$ & $1.9 \times 10^{-3}$ & 6.70 \\
\hline $\mathrm{NG}(0.5) 20$ & $1.5 \times 10^{-5}$ & $1.6 \times 10^{-3}$ & $1.6 \times 10^{-5}$ & $1.8 \times 10^{-3}$ & 6.62 \\
\hline
\end{tabular}



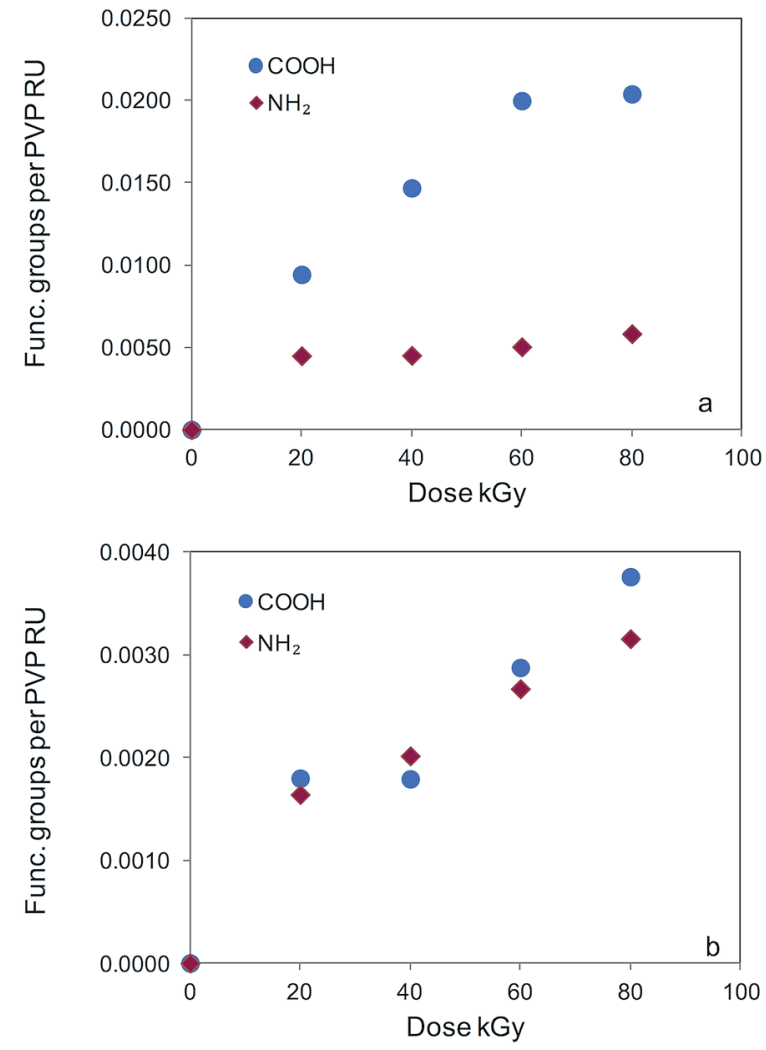

Fig. 9 (a and b) Number of carboxyl and primary amino groups formed on PVP nanogels irradiated at $0.1 \mathrm{wt} \%$ (a) and $0.5 \mathrm{wt} \%$ (b) as function of the irradiation dose.

The pH-values of the irradiated solutions measured after dialysis (prior to the titrations) also reflect the functional group imbalance observed for the low polymer concentration, the most acidic solutions being those of systems exposed to high doses where the imbalance in favor of the acid functionality is most pronounced.

Admittedly, the exact mechanisms behind the $\mathrm{NH}_{2^{-}}$and $\mathrm{COOH}$-group formations are unknown. However, it is obvious that $\mathrm{NH}_{2}$-groups can only be formed as a consequence of double $\mathrm{N}-\mathrm{C}$ bond scission of the pyrrolidone ring and that $\mathrm{COOH}^{-}$ groups can only be formed upon oxidative scission of the polymer backbone or the pyrrolidone rings.

An interesting observation is the fact that the particle concentration is lower in the $0.5 \%$ PVP-system than in the $0.1 \%$ PVP-system above $20 \mathrm{kGy}$. As seen both in the simulations and the experiments presented above, $\mathrm{H}_{2} \mathrm{O}_{2}$-formation increases with decreasing hydroxyl radical scavenger concentration.

Consequently, the $\mathrm{H}_{2} \mathrm{O}_{2}$-formation is expected to be higher in the $0.5 \%$ PVP-system than in the $0.1 \%$ PVP-system at doses above $20 \mathrm{kGy}$. It is therefore tempting to assume that the observed limitation in $\mathrm{NH}_{2}$-group formation at the lower polymer concentration can be attributed to lower production of $\mathrm{H}_{2} \mathrm{O}_{2}$ in the $0.1 \%$ PVP-system.

This would imply that the $\mathrm{C}-\mathrm{N}$ bond scission required for $\mathrm{NH}_{2}$-formation is facilitated by the presence of $\mathrm{H}_{2} \mathrm{O}_{2}$ or the subsequently formed $\mathrm{O}_{2}$. The most probable macroradical reactant for this reaction is an $\alpha$-amino alkyl radical capable of reducing both $\mathrm{H}_{2} \mathrm{O}_{2}$ and $\mathrm{O}_{2}$.

\section{Conclusions}

Pulsed e-beam irradiation constitutes a versatile, "green", onepot synthesis route for the production of multifunctional biocompatible nanogels. Irradiation can be performed with industrial accelerators, typical set-ups and doses applied for sterilization; therefore it may not even represent an additional cost in the production process. Size, molecular weight and functionalization are controlled by dose rate, polymer concentration and total dose without adding any other chemicals but $\mathrm{H}_{2} \mathrm{O}$ and PVP. The polymer itself acts as a template and product purification is straightforward and relatively inexpensive.

Under conditions favoring the formation of nanogels (i.e. low polymer concentration), significant amount of $\mathrm{H}_{2} \mathrm{O}_{2}$ is produced. This by-product appears to be key to functionalization. It should be appreciated that the possibility of formation of carboxyl groups and primary amino groups, reported here for the first time, transforms the otherwise chemically inert polymer into a functionalized nanocolloid amenable of decoration with (bio)molecules and cell receptors-specific ligands of therapeutic and/or diagnostic relevance. Finally, this work demonstrates that starting from aqueous solutions of the polymer, it is possible to fine-tune the functional group density of the nanogels independently from size and molecular weight.

\section{Acknowledgements}

The authors are grateful to Prof. Mohamad Al-Sheikhly and Eddie Salgado at the Department of Materials Science and Engineering, A. J. Clark School of Engineering, University of Maryland for the experimental support with liquid AFM. This research was partially supported by a grant from the Italian Ministry of University and Scientific Research (PRIN 2010-2011 - NANOMED 2010FPTBSH), Università degli Studi di Palermo FFR2012/2013, and by the International Atomic Energy Agency (IAEA) Coordinated Research Project (CRP F22064) "Nano sized delivery systems for radiopharmaceuticals" - Research Agreement No. 18349/R0.

\section{References}

1 A. Charlesby, Irradiation Effects on Polymers, ed. D. W. Clegg. and A. A. Collyer, Elsevier Applied Science, New York, 1991.

2 A. Chapirò, Radiation chemistry of polymeric systems, IV General Aspects of Radiation-Initiated Polymerization, WileyInterscience, New York, 1962.

3 P. Ulanski and J. M. Rosiak, Nucl. Instrum. Methods Phys. Res., Sect. B, 1999, 151, 356-360.

4 J. M. Rosiak and P. Ulanski, Radiat. Phys. Chem., 1999, 55, 139-151.

5 J. M. Rosiak, I. Janik, S. Kadłubowski, M. Kozicki, P. Stasica and P. Ulanski, Nucl. Instrum. Methods Phys. Res., Sect. B, 2003, 208, 325-330. 
6 S. Kadlubowski, J. Grobelny, W. Olejniczak, M. Cichomski and P. Ulanski, Macromolecules, 2003, 36, 2484-2492.

7 J. K. Jeszka, S. Kadlubowski and P. Ulanski, Macromolecules, 2006, 39(2), 2857-2870.

8 J. C. An, A. Weaver, B. Kim, A. Barkatt, D. Poster, W. N. Vreeland, J. Silverman and M. Al-Sheikhly, Polymer, 2011, 52, 5746-5755.

9 S. Kadlubowski, P. Ulanski and J. M. Rosiak, Polymer, 2012, 53, 1985-1991.

10 C. Dispenza, M. A. Sabatino, N. Grimaldi, D. Bulone, M. L. Bondi, M. P. Casaletto, S. Rigogliuso, G. Adamo and G. Ghersi, Biomacromolecules, 2012, 13, 1805-1817.

11 M. A. Sabatino, D. Bulone, M. Veres, A. Spinella, G. Spadaro and C. Dispenza, Polymer, 2013, 54(1), 154-164.

12 R. T. Chacko, J. Ventura, J. Zhuang and S. Thayumanavan, Adv. Drug Delivery Rev., 2012, 64, 836-851.

13 K. Raemdonck, J. Demeester and S. De Smedt, Soft Matter, 2009, 5(4), 4707-4715.

14 N. A. Peppas, J. Z. Hilt, A. Khademhosseini and R. Langer, Adv. Mater., 2006, 18, 1345-1360.

15 S. V. Vinogradov, Nanomedicine, 2010, 5(2), 2165-2168.

$16 \mathrm{H}$. A. Abd El-Rehim, A. E. Swilem, A. Klingner, El-S. A. Hegazy and A. A. Hamed, Biomacromolecules, 2013, 14(3), 3688-3698.

17 G. Adamo, N. Grimaldi, S. Campora, M. A. Sabatino, C. Dispenza and G. Ghersi, Chem. Eng. Trans., 2014, 38, 457-462.

18 C. Dispenza, G. Adamo, M. A. Sabatino, N. Grimaldi, D. Bulone, M. L. Bondi, S. Rigogliuso and G. Ghersi, J. Appl. Polym. Sci., 2014, 131(2), 239774-239780.

19 P. Picone, L. A. Ditta, M. A. Sabatino, V. Militello, P. L. San Biagio, M. L. Di Giacinto, L. Cristaldi, D. Nuzzo, C. Dispenza, D. Giacomazza and M. Di Carlo, Biomaterials, 2016, 80, 179-194.

20 S. Pardo, C. Dispenza, M. A. Sabatino, N. Grimaldi, A. Ajovalasit, L. Ditta and M. Jonsson, Gadolinium-chelating nanogels as MR contrast agents specifically targeting tumor cells ECR, 2014, DOI: 10.1594/ecr2014/c-0556.

21 R. J. Woods and A. K. Pikaev, Applied Radiation Chemistry: Radiation Processing, John Wiley and Sons Inc., New York, 1994, ch. 6.

22 N. Bartoszek, P. Ulanski and J. M. Rosiak, Int. J. Chem. Kinet., 2011, 43(9), 9474-9481.

23 W. L. McLaughlin, A. W. Boyd, K. H. Chadwick, J. C. McDonald and A. Miller, Dosimetry for radiation processing, Taylor and Francis, London, 1989.

24 J. A. Ghormley and A. C. Stewart, J. Am. Chem. Soc., 1956, 78, 2934-2939.

25 C. J. Hochanadel, J. Phys. Chem., 1952, 56, 587-594.

26 M. B. Carver, D. V. Hanley and K. R. Chaplin, MAKSIMAChemist a program for mass action kinetics simulation by automatic chemical equation manipulation and integration using stiff techniques, Tech. Rep. Atomic Energy of Canada Limited - Chalk River Nuclear Laboratories, Ontario, 1979.

27 P. Stepanek, Data Analysis in Dynamic Light Scattering, in Dynamic Light Scattering: The Method and Some Applications, ed. W. Brown, Oxford University Press, Oxford, U.K, 1993, pp. 177-240.

28 D. E. Koppel, J. Chem. Phys., 1972, 57, 4814-4820.

29 Y. L. Lyubchenko, J. Biomol. Struct. Dyn., 1992, 10(3), 35893606.

30 Y. Chen and Y. Zhang, Anal. Bioanal. Chem., 2011, 399, 25032509.

31 A. Hennig, A. Hoffmann, H. Borcherding, T. Thiele, U. Schedler and U. Resch-Genger, Anal. Chem., 2011, 83(12), 124970-124974.

32 P. Jain and S. H. Yalkowsky, Int. J. Pharm., 2007, 342, 1-5.

33 R. M. Silverstein, G. C. Bassler and T. C. Morrill, Spectrometric identification of organic compounds, John Wiley \& Sons Inc, New York, 3rd edn 1974. 